\title{
BEST APPROXIMATION AND FIXED POINTS IN STRONG M-STARSHAPED METRIC SPACES
}

\author{
M. A. AL-THAGAFI \\ Department of Mathematics \\ Fing Abdul Aziz University \\ P. O. Box 30608. Jeddah 21487. Saudi Arabia
}

(Received January 11, 1994)

\begin{abstract}
We introduced strong $M$-starshiped metric spaces. For these spaces, we obtained two fixed-point theorems generalizing a result of W. G. Dotson, and two theorems extending and subsuming several known results on the existence of fixed points of best approximation.
\end{abstract}

KEY WORDS AND PHRASES. Best approximation. strong $M$-starshaped metric spaces, fixed points.

1991 AMS SUBJECT CLASSIFICATION CODES. 41A65, 54H25, 47H10.

\section{INTRODUCTION.}

Let $X$ be a metric space, $T: X \rightarrow X . K$ and $D$ subsets of $X$, and $p \in X . T$ is $\gamma$-nonexpansive on $D$ if $d(T x, T y) \leq d(x, y)$ for every $x, y \in D$ with $d(x, y) \leq \gamma . \quad T$ is $\gamma$-contraction on $D$ if $d(T x, T y) \leq \lambda d(x, y)$ for some $\lambda \in[0,1)$ and for every $x, y \in D$ with $d(x, y) \leq \gamma . \quad X$ is a $\gamma$-chainable metric space if for any pair $x, y \in X$, there exists a finite chain of points $x_{0}, x_{1}, \cdots, x_{n-1}, x_{n}$ in $X$ with $x_{0}=x$ and $x_{n}=y$ such that $d\left(x_{1}-1, x_{1}\right) \leq \gamma$ for $\imath=1,2, \cdots, n$. The set of best $K$ approximations to $p$, denoted by $B_{K}(p)$, is the set of all $x \in K$ such that $d(x, p)=\delta(p, K)$, where $\delta(p, K)=\inf _{z \in K} d(z, p)$.

Brosowski [1] proved that if $X$ is a nomed linear space, $T: X \rightarrow X$ is nonexpansive with a fixed point $p, K \subset X$ with $T(K) \subset K$, then $T$ lias a fixed point in $B_{K}(p)$ provided that $B_{K}(p)$ is nonempty, compact and convex. Singh ([6], Theorem 1) relaxed the convexity of $B_{K}(p)$ be starshapedness. However, Hicks and Humphries ([4], p. 221) showed that the conclusion of Singh's result still holds whencver $T(K) \subset K$ is replaced by $T(\partial K) \subset K$. Subrahmanyam ([8], Theorem 3) proved that if $X$ is a normed linear space, $T: X \rightarrow X$ is $\delta(p, K)$-nonexpansive with a fixed point $p, K \subset X$ with $T(K) \subset K$, then $T$ has a fixed point in $B_{K}(p)$ provided that $K$ is a finite-dimensional subspace of $X$. However, Singh ([7], Theorem 1) showed that the conclusion of Subrahmanyam's result still holds whenever the finite-dimensionality of $K$ is replaced by the following conditions:

(i) $B_{K}(p)$ is nonempty, compact and starshaped, and

(ii) $T$ is continuous on $B_{K}(p)$.

Recently, Sahab and Than ([5]. Theorem 3.1) showed that Singh's second result still holds whenever $X$ is a strong convex metric space (see Definition 3.1 below).

Our aim, in this paper, is to establish results extending and subsumnng the above best approximation results. To do this. we introduce. $I$-starshaped and strong $M$-starshaped metric 
spaces. Convex and starshaped metric spaces of Takahashi [9] are examples of $\boldsymbol{M}$-starshaped metric spaces. Then we obtained two fixed-point theorems generalizing a result of $\mathrm{W}$. G. Dotson. We prove the first using a result of M. Edclstein and the second using Banach's contraction principle. Using the first theorem, we established our results on the existence of fixed points of best approximation.

For later use, we state the following result of M. Edelstein ([3], Theorem 5.2).

THEOREM 1.1. Let $D$ be a complete and $\gamma$-chainable metric space. If $S: D \rightarrow D$ is $\gamma$ contraction, then $S$ has a unique fixed point in $D$.

\section{FIXED POINTS IN STRONG $M$-STARSHAPED METRIC SPACES.}

DEFINITION 2.1. Let $X$ be a metric space. $M \subset X$ and $I=[0,1]$.

(a) $X$ is $M$-starshaped if there exists a mapping $W: X \times M \times I \rightarrow X^{-}$satisfying

$$
d(x, W(y, q, \lambda)) \leq \lambda d(x, y)+(1-\lambda) d(x, q)
$$

for every $x, y \in X$, all $q \in M$ and all $\lambda \in I$.

(b) $X$ is strong $M$-starshaped if it is $M$-starshaped and $W$ satisfies

$$
d(W(x, q, \lambda), W(y, q, \lambda)) \leq \lambda d(x, y)
$$

for every $x, y \in X$, all $q \in M$ and all $\lambda \in I$.

(c) $X$ is (strong) convex if it is (strong) $X$-starshaped. $X$ is starshaped if it is $\{q\}$ starshaped for some $q \in X$.

Convex and starshaped metric spaces were introduced by Takahashi [9]. Each normed linear space $X$ is a strong convex metric space with $W$ defined by $W(x, q, \lambda)=\lambda x+(1-\lambda) q$ for every $x, q \in X$ and all $\lambda \in I$.

DEFINITION 2.2. Let $X$ be a $M$-starshaped metric space. A subset $D$ of $X$ is $q$-starshaped if there exists $q \in D \cap M$ with $W(D \times\{q\} \times I) \subset D$. A $q$-starshaped subset of a convex metric space is called starshaped.

THEOREM 2.3. Let $X$ be a strong $M$-starshaped metric space and $D \subset X$. If $D$ is compact, $\gamma$-chainable and $q$-starshaped, and $T: D \rightarrow D$ is $\gamma$-nonexpansive, then $T$ has a fixed point in $D$.

PROOF. For each positive integer $n$, let $\lambda_{n}=\frac{n}{n+1}$ and $T_{n} x=W\left(T x, q, \lambda_{n}\right)$ for all $x \in D$. By the $\gamma$-nonexpansiveness of $T$ on $D$, each $T_{n}$ satisfies

$$
d\left(T_{n} x, T_{n} y\right)=d\left(W\left(T x, q, \lambda_{n}\right), W\left(T y, q, \lambda_{n}\right)\right) \leq \lambda_{n} d(T x, T y) \leq \lambda_{n} d(x, y)
$$

for every $x, y \in D$ with $d(x, y) \leq \gamma$. Note that $D$ is $q$-starshaped and $T: D \rightarrow D$. So each $T_{n}$ is a $\gamma$ contraction selfmap of $D$. Since $D$ is $\gamma$-chainable, Theorem 1.1 shows that each $T_{n}$ has a unique fixed point $x_{n} \in D$. By the compactness of $D$, there exists a subsequence $\left\{x_{n_{1}}\right\}$ of $\left\{x_{n}\right\}$ with $\lim _{\imath \rightarrow \infty} x_{n_{t}}=x_{0} \in D$. Since

$$
d\left(T x_{n_{i}}, x_{n_{\mathfrak{z}}}\right)=d\left(T x_{n_{i}}, W\left(T x_{n_{i}}, q, \lambda_{n_{\mathfrak{z}}}\right)\right) \leq\left(1-\lambda_{n_{\mathfrak{i}}}\right) d\left(T x_{n_{\mathfrak{i}}}, q\right)
$$

for all $i$, then $\lim _{\imath \rightarrow \infty} d\left(T x_{n_{z}}, x_{n_{z}}\right)=0$. Now, the $\gamma$-nonexpansiveness of $T$ on $D$ implies its continuity, and hence $x_{0}$ is a fixed point of $T$.

The following is a result of Dotson ([2], Theorem 1).

COROLLARY 2.4. Let $X$ be a normed linear space and $D \subset X$. If $D$ is compact and starshaped and if $T: D \rightarrow D$ is nonexpansive, then $T$ has a fixed point in $D$. 
PROOF. For every $\gamma>0 . D$ is $\gamma$-chaind le and $T$ is $\gamma$-nonexpansive.

THEOREM 2.5. Let $X$ be a strong $M$-stanshaped metric space and $D \subset \mathrm{X}$. If $D$ is compact and $q$-starshaped and if $T: D \rightarrow D$ is nonexpansive, then $T$ has a fixed point in $D$.

The proof of Theorem 2.5 is similar to the one given for Theorem 2.3; however, we use Banach's contraction principle instead of Theosem 1.1. Note that Corollary 2.4 follows also from Theorem 2.j.

\section{BEST APPROXIMATION IN STRONG $M$-STARSHAPED METRIC SPACES.}

LEMMA 3.1. Let $X$ be a strong $M$-starshaped metric space, $K \subset X$ and $p \in X$. If $B_{K}(p)$ is $q$-starshaped, then $B_{K}(p)$ is $\delta\left(p, K^{*}\right)$-chainable.

PROOF. For $x, y \in B_{K}(p)$, let

$$
x_{0}=x, \quad x_{1}=\amalg\left(x, q, \frac{1}{2}\right), x_{2}=\amalg\left(y, q, \frac{1}{2}\right), x_{3}=y .
$$

Since $B_{K}(p)$ is $q$-starshaped. then $x_{0}, x_{1}, x_{2}, x_{3}$ belong to $B_{K}(p)$. Now, the strong $\boldsymbol{M}$ starshapedness of $X$ implies that

$$
\begin{aligned}
& d\left(x_{0}, x_{1}\right)=d\left(x, W\left(x, q \cdot \frac{1}{2}\right)\right) \leq \frac{1}{2} d(x, q) \leq \delta(p, K), \\
& d\left(x_{1}, x_{2}\right)=d\left(W\left(x, q, \frac{1}{2}\right), W\left(y, q, \frac{1}{2}\right)\right) \leq \frac{1}{2} d(x, y) \leq \delta(p, K), \\
& d\left(x_{2}, x_{3}\right)=d\left(W\left(y, q, \frac{1}{2}\right), y\right) \leq \frac{1}{2} d(y, q) \leq \delta(p, K) .
\end{aligned}
$$

Therefore $B_{K}(p)$ is $\delta(p, K)$-chainable.

LEMMA 3.2. Let $X$ be a $M$-starshaped metric space, $K \subset X$ and $p \in X$. Then $B_{K}(p) \subset \partial K \cap K$.

PROOF. Let $y \in B_{K}(p)$ and let $\lambda_{n}=\frac{n}{n+1}$ for each positive integer $n$. Then

$$
d\left(p, W\left(y, p, \lambda_{n}\right)\right) \leq \lambda_{n} d(p, y)<\delta(p, K)
$$

which implies that $W\left(y, p, \lambda_{n}\right) \notin K$ for every $n$. Since

$$
d\left(y, W\left(y, p, \lambda_{n}\right)\right) \leq\left(1-\lambda_{n}\right) d(y, p)=\left(1-\lambda_{n}\right) \delta(p, K)
$$

for all $n$, then $\lim _{n \rightarrow \infty} W\left(y, p, \lambda_{n}\right)=y$. So each neighborhood of $y$ contains at lcast one $W\left(y, p, \lambda_{n}\right)$, hence $y \in \partial K$.

THEOREM 3.3. Let $X$ be a strong $M$-starshaped metric space, $T: X \rightarrow X, K \subset X$, and $p \in X$ a fixed point of $T$. If $B_{K}(p)$ is compact and $q$-starshaped, $T(K) \subset K$, and $T$ is $\delta(p, K)$ nonexpansive on $B_{K}(p) \cup\{p\}$, then $T$ has a fixed point in $B_{K}(p)$.

PROOF. Let $y \in B_{K}(p)$. Then $T y \in K$ and, by the $\delta(p . K)$-nonexpansiveness of $T$ on $B_{K}(p) \cup\{p\}, d(T y, p) \leq d(y, p)$. Thus $T y \in B_{K}(p)$ and so $T: B_{K}(p) \rightarrow B_{K}(p)$. Now, Theorem 2.3, with $D=B_{K}(p)$, and Lemma 3.1 show that $T$ has a fixed point in $B_{K}(p)$.

THEOREM 3.4. Let $X$ be a strong $M$-starshaped metric space, $T: X \rightarrow X, K \subset X$, and $p \in M$ a fixed point of $T$. If $B_{K}(p)$ is compact and $q$-starshaped, $T(\partial K \cap K) \subset K$, and $T$ is $\delta(p, K)$-nonexpansive on $B_{K}(p) \cup\{p\}$, then $T$ has a fixed point in $B_{K}(p)$.

PROOF. Let $y \in B_{K}(p)$. Then $y \in \partial K \cap K$ by Lemma 3.2. Since $T(\partial K \cap K) \subset K$, then $T y \in K$. Now, the $\delta(p, K)$-nonexpansiveness on $B_{K}(p) \cup\{p\}$ implies that $T y \in B_{K}(p)$. Therefore $T: B_{K}(p) \rightarrow B_{K}(p)$. Now, Theorem 2.3, with $D=B_{K}(p)$, and Lemma 3.1 show that $T$ has a fixed point in $B_{K}(p)$. 
COROLLARY 3.5. Let $X$ be a strong convex metric space, $T: X \rightarrow X, K \subset X$, and $p \in X$ a fixed point of $T$. If $B_{K}(p)$ is compact and starshaped, $T(\partial K \cap K) \subset K$. and $T$ is $\delta(p, K)$ nonexpansive on $B_{K}(p) \cup\{p\}$, then $T$ has a fixed point in $B_{K}(p)$.

All best approximation results mentioned in section 1 follow from Corollary 3.5. Moreover, if $T\left(\partial K^{\circ} \cap K^{\circ}\right) \subset K^{\prime}$ is replaced by $T\left(K^{\circ} \subset K^{\prime}\right.$ in Conollary 3.5. we will have Salab and Khan result ([5], Theorem 3.1) and it will follow from Theorem 3.3. Note that their assumption for the continuity of $T$ on $B_{K}(p)$ is implied by the $\delta(p, K)$-nonexpansiveness of $T$ on $B_{K}(p) \cup\{p\}$.

\section{REFERENCES}

1. BROSOWSKI, B., Fixpunktsätze in der approximations-theorie, Mathematıca (Cluj) 11 (1969), 195-220.

2. DOTSON, JR., W.G., On fixed points of nonexpansive mappings in nonconvex sets, Proc. Amer. Math. Soc. 38 (1973), 155-156.

3. EDELSTEIN, M., An extension of Banach's contraction principle, Proc. Amer. Math. Soc. $12(1961), 7-10$.

4. HICKS, T.L. and HUMPHRIES, M.D., A note on fixed-point theorems, J. Approx. Theory 34 (1982), 221-225.

5. SAHAB, S.A. and KHAN, M.S., Best approximation in spaces with convex structure, Bull. Inst. Math. Acad. Sinica 17 (19S9), 59-63.

6. SINGH, S.P., An application of a fixed point theorem to approximation theory, J. Approx. Theory 25 (1979), 89-90.

7. SINGH, S.P., Applications of fixed point theorems in approximation theory, Applied Nonlinear Analysis (Ed., V. Lakshmikantham), Academic Press, New York (1979), 389-397.

8. SUBRAHMANYAM, P.V., An application of a fixed point theorem to best approximation, J. Approx. Theory 20 (1977), 165-172.

9. TAKAHASHI, W., A convexity in metric spaces and nonexpansive mappings, Kodai Math. Sem. Rep. $22(1970), 142-149$. 


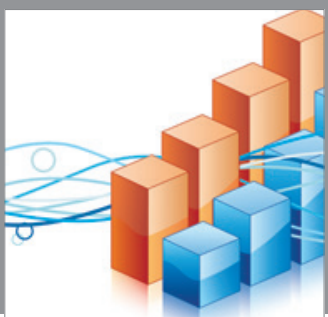

Advances in

Operations Research

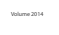

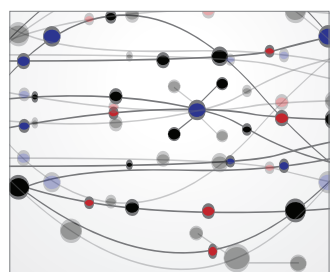

\section{The Scientific} World Journal
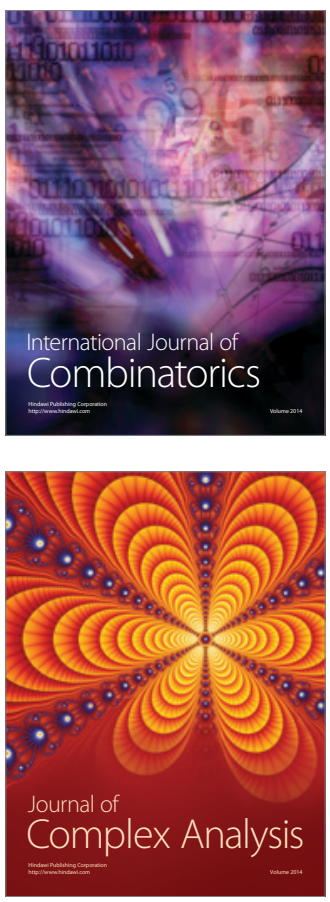

International Journal of

Mathematics and

Mathematical

Sciences
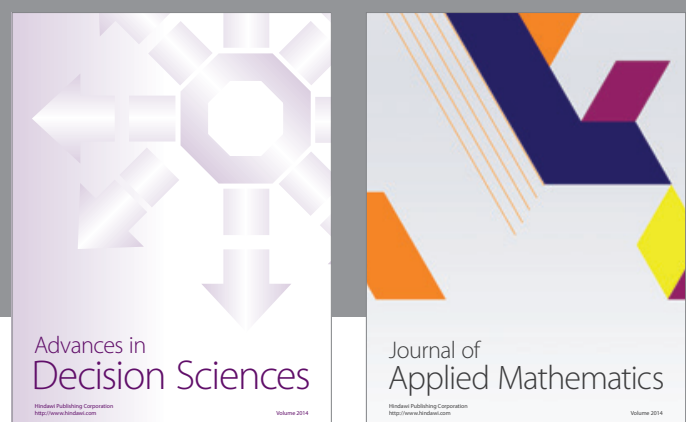

Journal of

Applied Mathematics
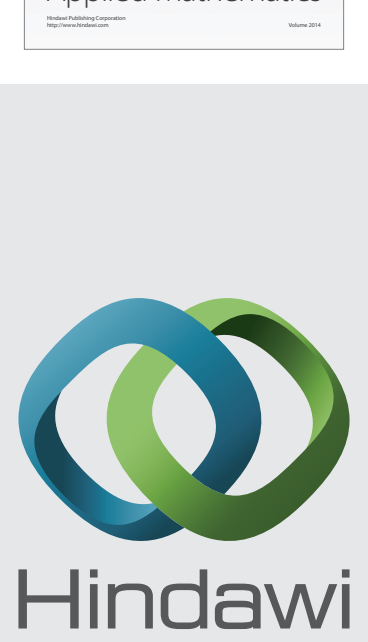

Submit your manuscripts at http://www.hindawi.com
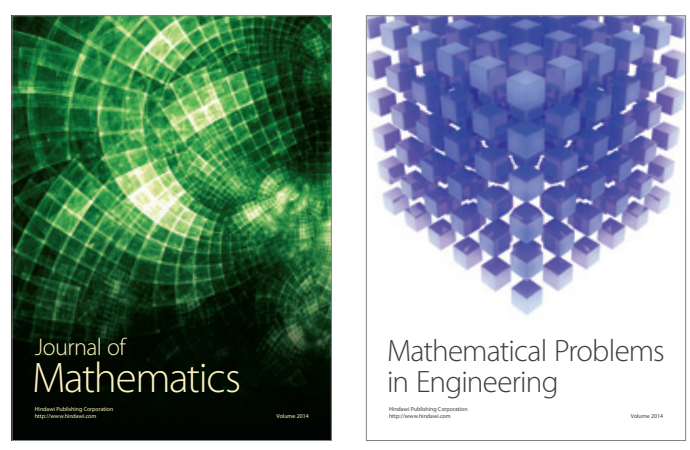

Mathematical Problems in Engineering
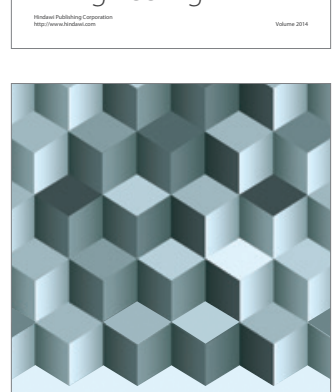

Journal of

Function Spaces
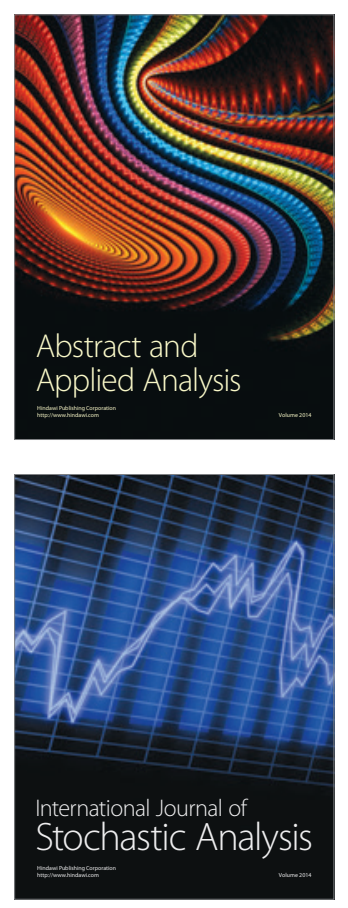

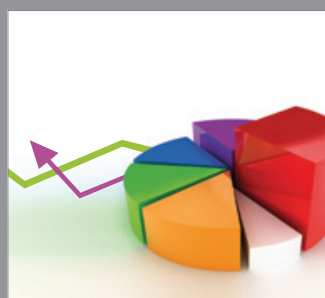

ournal of

Probability and Statistics

Promensencen
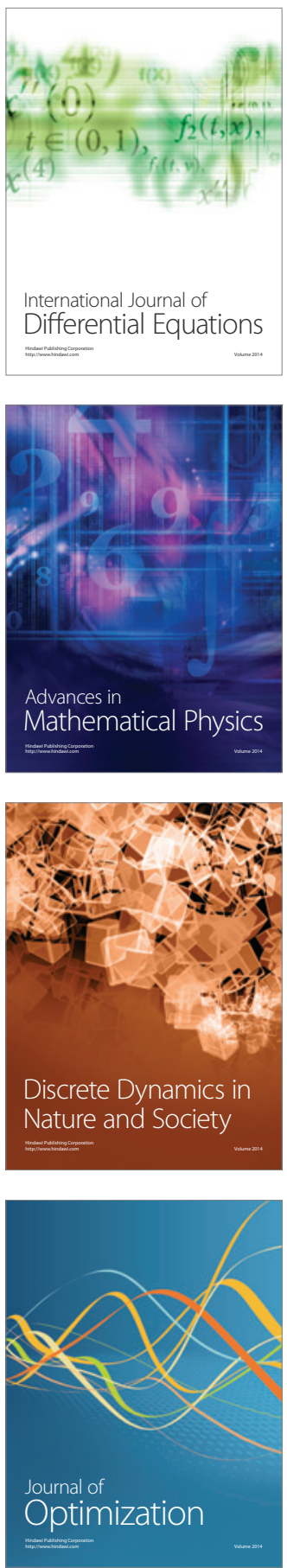